\title{
S-1 and S-2-alar-iliac screw fixation via intraoperative navigation
}

\author{
Martin H. Pham, MD, ${ }^{1}$ Andre M. Jakoi, MD, ${ }^{2}$ and Patrick C. Hsieh, MD1 \\ Departments of ${ }^{1}$ Neurosurgery and ${ }^{2}$ Orthopaedic Surgery, Keck School of Medicine, University of Southern California, \\ Los Angeles, California
}

Adult deformity patients often require fixation to the sacrum and pelvis for construct stability and improved fusion rates. Although certain sacropelvic fixation techniques can be challenging, the availability of intraoperative navigation has made many of these techniques more feasible. In this video case presentation, the authors demonstrate the techniques of S-1 bicortical screw and S-2-alar-iliac screw fixation under intraoperative navigation in a 67-year-old female. This instrumentation placement was part of an overall T-10-pelvis construct for the correction of adult spinal deformity.

The video can be found here: https://youtu.be/3HZo-80jQr8.

KEYWORDS intraoperative navigation; S-2-alar-iliac; S2Al; sacroiliac fixation; video 